\title{
Biospecimens Not Retained
}

National Cancer Institute

\section{Source}

National Cancer Institute. Biospecimens Not Retained. NCI Thesaurus. Code C127776.

An indication that no biospecimen samples were retained as reserve samples. 